\title{
1 Glutathione is a highly efficient thermostabilizer of poliovirus Sabin strains
}

2 Running title: Thermostabilization of poliovirus by glutathione

$3 \quad$ Rana Abdelnabi, Leen Delang, Johan Neyts

4 KU Leuven - University of Leuven, Department of Microbiology and Immunology, Rega

5 Institute for Medical Research, Laboratory of Virology and Chemotherapy, B-3000

6 Leuven, Belgium.

7

8 *Corresponding author: Johan Neyts, Heresstraat 49, 3000 Leuven, Belgium. Tel 9 +3216321105, email: Johan.Neyts@kuleuven.be. 
Abstract

Glutathione (GSH) is the most abundant thiol peptide in animal cells and has a critical role in antioxidation. GSH was reported to be essential for stabilization of some enteroviruses, including poliovirus (PV), during viral morphogenesis. Here, we explored the potential use of GSH as a thermostabilizer of oral poliomyelitis vaccine (OPV) formulations. GSH significantly protected the three types of PV from heat-inactivation in a concentrationdependent manner. At a GSH concentration of $20 \mathrm{mM}$, nearly complete protection was observed against heating temperatures up to $53^{\circ} \mathrm{C}$ for $2 \mathrm{~min}$. GSH also markedly protected PV1 from heat-inactivation and this up to 6 hours at temperatures of $44^{\circ} \mathrm{C}$ and $46^{\circ} \mathrm{C}$ and 3 hours at $48^{\circ} \mathrm{C}$. The fact that GSH is naturally present at high concentration in the human body makes it an efficient candidate stabilizer for OPV formulations.

\section{Key words}

Glutathione; poliomyelitis; cold chain; vaccine; Sabin 
25 - Glutathione (GSH) is an essential host factor for stabilization of poliovirus during viral 26 morphogenesis.

27 - Glutathione increased the thermostability of poliovirus Sabin strains in a 28 concentration-dependent manner.

29 - The thermostabilization by GSH was also observed after prolonged periods of 30 incubation at temperatures up to $50^{\circ} \mathrm{C}$. 
Glutathione (GSH) is the most abundant thiol peptide in animal cells, it has a critical role in antioxidation, detoxification of xenobiotics and maintenance of immune system functions [1-3]. GSH is a tripeptide composed of glutamine, cysteine and glycine, which is readily oxidized into the disulfide form (GSSG) in a non-enzymatic reaction [2]. The imbalance of the ratio of reduced and oxidized forms of glutathione (GSH/GSSG) is observed in several human diseases such as cancer, neurodegenerative diseases and some viral infection such as those caused by HIV-1 $[2,4-6]$. Our laboratory demonstrated earlier that GSH is an essential host factor for stabilization of Coxsackieviruse B3 (CVB3), an enterovirus, during viral morphogenesis. Selective and rapid depletion of intracellular GSH level results in a potent anti-enterovirus effect [7]. Enteroviruses C, including poliovirus, also require GSH during their morphogenesis [8].

The oral polio vaccine (OPV) has been widely and successfully used in the poliomyelitis eradication programs. Because of its sensitivity to temperature, this live-attenuated vaccine needs a strict cold-chain for storage and transport [9]. Several strategies have been explored to increase the thermostability of OPV such as the use of $\mathrm{MgCl}_{2}$ [10], sucrose [11], heavy water (D20) [12], capsid binders [13] and fatty acids [14]. Currently, $\mathrm{MgCl}_{2}$ is the most widely used stabilizer for OPV formulations [15]. However, some OPV formulations are also stabilized with sucrose (35-50\%) [15].The half-life of stabilized OPV is $48 \mathrm{~h}$ at $37^{\circ} \mathrm{C}, 24 \mathrm{~h}$ at $41^{\circ} \mathrm{C}, 14 \mathrm{~h}$ at $45^{\circ} \mathrm{C}$ and $3 \mathrm{~h}$ at $50^{\circ} \mathrm{C}[9,15]$. Here, we wanted to explore the effect of GSH on the thermostability of different types of PV vaccine strains and thus the possible use of GSH as a new stabilizer for oral poliovirus vaccine (OPV) formulation. This may allow manipulation of OPV out of the cold-chain for longer periods of time which will reduce the cost for vaccine transport, especially in non-developed countries. First, to explore whether GSH has the potential to serve as a thermostabilizier for the oral polio vaccine, the dose-response effect of GSH on the thermostability of the three types of 
poliovirus Sabin strains (PVs) was studied. Briefly, PV1, PV2 and PV3 (TCID50/ml= 2x106, $2 \times 10^{5}$ and $1.4 \times 10^{5}$, respectively) were incubated with different GSH concentrations (2-50 $\mathrm{mM}$, prepared in PBS) for $30 \mathrm{~min}$ at $48^{\circ} \mathrm{C}$. The three types of PV were markedly protected from heat-inactivation in a concentration-dependent manner (Fig.1). At a concentration of $20 \mathrm{mM}$ or higher, GSH resulted in near complete protection of the infectivity of the three strains. For this reason, $20 \mathrm{mM}$ of GSH was selected for subsequent studies (Fig.1). The $\mathrm{pH}$ of GSH solutions of $20 \mathrm{mM}, 10 \mathrm{mM}, 5 \mathrm{mM}$ and $2 \mathrm{mM}$ is respectively 3.6; 4.0; 6.2 and 6.6. As shown in (Fig.1), the stabilizing effect of $5 \mathrm{mM} \mathrm{GSH}$ solution on PV1 and PV2 is comparable to that of 10 and $20 \mathrm{mM} \mathrm{GSH}$ solutions. It thus appears that the thermostabilizing effect of GSH is $\mathrm{pH}$ independent and that this stabilizing effect is mainly due to the direct interaction of the molecule with the viral capsid [7]. The level of GSH in erythrocytes of infants is about $9 \mathrm{nmol} / \mathrm{mg}$ hemoglobin (Hb) [16]. This corresponds to $\sim 130 \mathrm{mg}$ total GSH in the blood $(\sim 280 \mathrm{ml})$ of a $4 \mathrm{~kg}$ weighing infant. Infants have also an intake of GSH via breastmilk. The average GSH concentration in breastmilk is $\sim 192$ $\mu \mathrm{mol} / \mathrm{L}(=59 \mathrm{mg} / \mathrm{L})$ [17]. Therefore, the concentration of GSH used in this study $(20 \mathrm{mM}$ or $6.2 \mathrm{mg} / \mathrm{ml}$ would correspond to $3.1 \mathrm{mg}$ per OPV dose which is equivalent to the GSH content in $53 \mathrm{ml}$ breastmilk. The effect of $20 \mathrm{mM} \mathrm{GSH}$ on the thermostability profile of PV was then determined by incubating PV1, PV2 and PV3 Sabin strains at different temperatures [range: 42 to $56^{\circ} \mathrm{C}$ ] for 2 min followed by rapid cooling at $4^{\circ} \mathrm{C}$. GSH markedly protected the 3 types of poliovirus vaccine strains from heat-inactivation at all the tested temperatures (Fig.2A-C). At the highest studied temperature $\left(56^{\circ} \mathrm{C}\right)$, GSH resulted in $100 \%$ protection of PV2 infectivity against heat-inactivation compared to the untreated control (Fig.2B). Also at $56^{\circ} \mathrm{C}$, GSH still resulted in a markedly protective effect where by 79\% of PV1 (Fig.2A) and 60\% of PV3 (Fig.2C) initial titers were recovered. 
Next, the time-dependency of the stabilizing effect of GSH was determined; to that end PV1 was incubated at either $44^{\circ} \mathrm{C}, 46^{\circ} \mathrm{C}, 48^{\circ} \mathrm{C}$ and $50^{\circ} \mathrm{C}$ over a time span of 6 hours in the presence or absence of $20 \mathrm{mM}$ GSH. Samples were collected every hour to quantify the remaining infectious viral load. GSH markedly protected PV1 from heat-inactivation for prolonged periods of time. Indeed, respectively 92 and $83 \%$ of the initial virus titers were recovered after 6 hours of incubation at $44^{\circ} \mathrm{C}$ (Fig.3A) and $46^{\circ} \mathrm{C}$ (Fig.3B). Whereas heating of the untreated PV1 for two hours at $48^{\circ} \mathrm{C}$ or one hour at $50^{\circ} \mathrm{C}$ resulted in a complete loss of the infectivity of the virus; 20 mM GSH resulted in 84 and $45 \%$ recovery of the initial PV1 titers following 3 hours of incubation at $48^{\circ} \mathrm{C}$ and $50^{\circ} \mathrm{C}$, respectively (Fig.3C/D).

In 1988, the Global Polio Eradication Initiative (GPEI) has been launched by WHO, these efforts reduced the number of PV cases by $>99 \%$ [18]. OPV has been widely used for polio eradication because of its lower cost, higher ability to induce intestinal immunity than the inactivated polio vaccine (IPV) and ease of administration. However, the use of OPV is associated with the risk of vaccine associated paralytic poliomyelitis (VAPP) due to development of mutants with the neurovirulence and transmission characteristics of the wild-type virus i.e. vaccine-derived polioviruses (VDPV) [19]. Therefore, the development of genetically-stable modified Sabin strains is currently under study to increase the safety of live attenuated polio vaccine. Recently, new PV2 Sabin strains have been developed and proven to be genetically-stable and hyper-attenuated [de Boer SM, Macadam A., Andino R., Burns C. and Bakker W. (Europic2016, Switzerland, 4 to 8 September 2016)]. Because of its safety and efficiency as a PV-thermostabilizer, GSH may serve as candidate stabilizer for such new OPV formulations. 


\section{Funding information}

104 This work was funded by a grant for the BELVIR project from BELSPO (IUAP) and the EU 105 FP7 Industry-Academia Partnerships and Pathways Project AIROPICO. L.D. was 106 supported by a post-doctoral fellowship from the FWO (Fund for Scientific Research of 107 Flanders, Belgium).

108

109 Conflict of interest: Non to declare

110 


\section{References}

112

[1] Fraternale A, Paoletti MF, Casabianca A, Nencioni L, Garaci E, Palamara AT, et al. GSH and analogs in antiviral therapy. Mol Aspects Med 2009;30:99-110. doi:10.1016/j.mam.2008.09.001.

[2] Lagman M, Ly J, Saing T, Singh MK, Tudela EV, Morris D, et al. Investigating the causes for decreased levels of glutathione in individuals with type II diabetes. PLoS One 2015;10. doi:10.1371/journal.pone.0118436.

[3] Marí M, Morales A, Colell A, García-Ruiz C, Fernández-Checa JC. Mitochondrial glutathione, a key survival antioxidant. Antioxid Redox Signal 2009;11:2685-700. doi:10.1089/ARS.2009.2695.

[4] Traverso N, Ricciarelli R, Nitti M, Marengo B, Furfaro AL, Pronzato MA, et al. Role of glutathione in cancer progression and chemoresistance. Oxid Med Cell Longev 2013. doi:10.1155/2013/972913.

[5] Morris D, Guerra C, Donohue C, Oh H, Khurasany M, Venketaraman V. Unveiling the mechanisms for decreased glutathione in individuals with HIV infection. Clin Dev Immunol 2012;2012. doi:10.1155/2012/734125.

[6] Johnson WM, Wilson-Delfosse AL, Mieyal JJ. Dysregulation of glutathione homeostasis in neurodegenerative diseases. Nutrients 2012;4:1399-440. doi:10.3390/nu4101399.

[7] Thibaut HJ, van der Linden L, Jiang P, Thys B, Canela MD, Aguado L, et al. Binding of Glutathione to Enterovirus Capsids Is Essential for Virion Morphogenesis. PLoS Pathog 2014;10. doi:10.1371/journal.ppat.1004039.

[8] Ma HC, Liu Y, Wang C, Strauss M, Rehage N, Chen YH, et al. An Interaction between Glutathione and the Capsid Is Required for the Morphogenesis of C-Cluster Enteroviruses. PLoS Pathog 2014;10. doi:10.1371/journal.ppat.1004052.

[9] Shrivastava A, Gupta N, Upadhyay P, Puliyel J. Caution needed in using oral polio vaccine beyond the cold chain: Vaccine vial monitors may be unreliable at high temperatures. Indian J Med Res 2012;135:520-2.

[10] Wallis C and Melnick JL. Stabilization of poliovirus by cations. Tex Rep Biol Med 1961;19:683-700.

[11] Mirchamsy H, Shafyi A, Mahinpour M, Nazari P. Stabilizing effect of magnesium chloride and sucrose on Sabin live polio vaccine. DevBiolStand 1978;41:255-7.

[12] Wu R, Georgescu MM, Delpeyroux F, Guillot S, Balanant J, Simpson K, et al. 
Thermostabilization of live virus vaccines by heavy water (D20). Vaccine 1995;13:1058-63. doi:10.1016/0264-410X(95)00068-C.

[13] Andries K, Rombaut B, Dewindt B, Boeyé a. Discrepancy between infectivity and antigenicity stabilization of oral poliovirus vaccine by a capsid-binding compound. J Virol 1994;68:3397-400.

[14] Dorval BL, Chow M, Klibanov AM. Stabilization of poliovirus against heat inactivation. Biochem Biophys Res Commun 1989;159:1177-83. doi:10.1016/0006-291X(89)92234-1.

[15] Milstien JB, Galazka AM, Kartoglu U, Zaffran M. Temperature sensitivity of vaccines. WHO Geneva 2006:1-58. doi:WHO/IVB/06.10.

[16] Lee Y, Chou Y. Antioxidant Profiles in Full Term and Preterm Neonates. Chang Gung Med J 2005:846-51.

[17] Ankrah N, Appiah-opong R, Dzokoto C. Human Breastmilk Storage and the Glutathione Content. J Trop Pediatr 2000:111-3.

[18] Moturi EK, Porter KA, Wassilak SGF, Tangermann RH, Diop OM, Burns CC, et al. Progress toward polio eradication - worldwide, 2013-2014. MMWR Morb Mortal Wkly Rep 2014;63:468-72. doi:10.1093/infdis/jit808.

[19] Orenstein WA, Committee on Infectious Diseases TCOI, Sutter R, Kew O, Cochi S, Aylward B, et al. Eradicating polio: how the world's pediatricians can help stop this crippling illness forever. Pediatrics 2015;135:196-202. doi:10.1542/peds.2014-3163. 


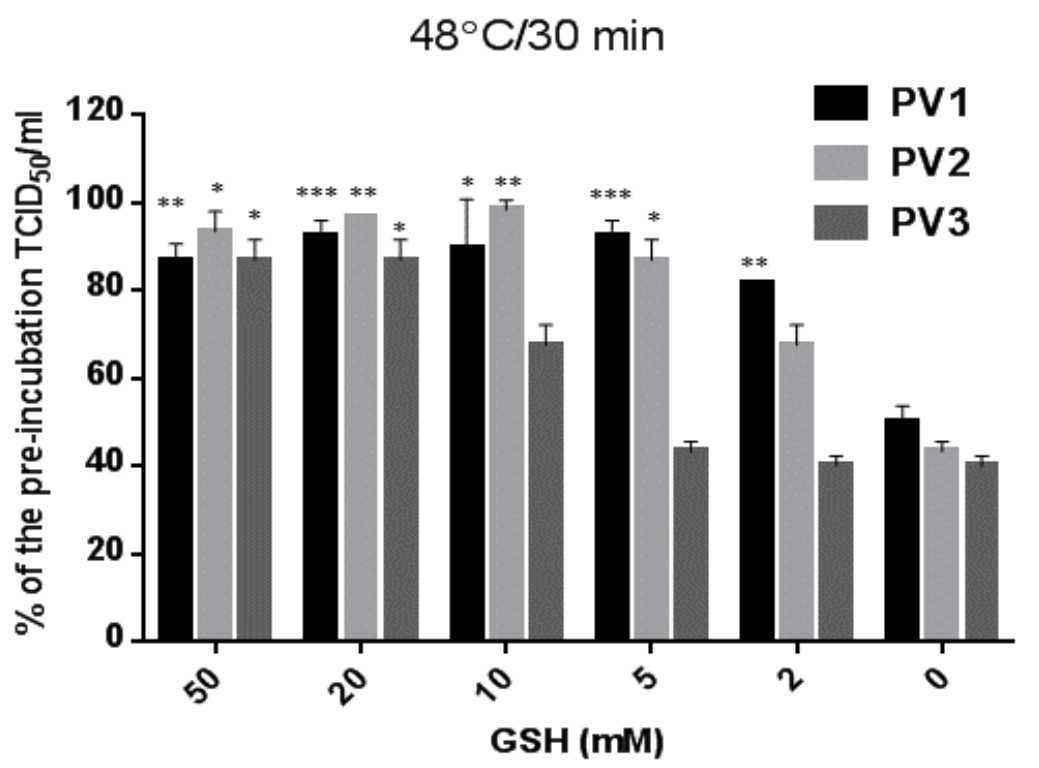

Fig.1. Concentration-dependent heat-stabilization of PVs by glutathione.

170 Polioviruses types 1, 2 and 3 were incubated with increasing concentrations of 171 glutathione (GSH) at $48^{\circ} \mathrm{C}$ for $30 \mathrm{~min}$, after which samples were stored on ice until the

172 remaining infectious viral load was quantified by endpoint titration and converted into \% 173 of the pre-incubation $\mathrm{TCID}_{50} / \mathrm{ml}$. The data presented are the average \pm SD of three 174 independent experiments. Significant differences between the control and GSH-treated 175 virus were assessed using the unpaired Student's t-test $\left({ }^{*} \mathrm{P}<0.05,{ }^{* *} \mathrm{P}<0.01,{ }^{* * *} \mathrm{P}=0.007\right)$. 176 Statistical analysis was performed using the Prism software (GraphPad). 

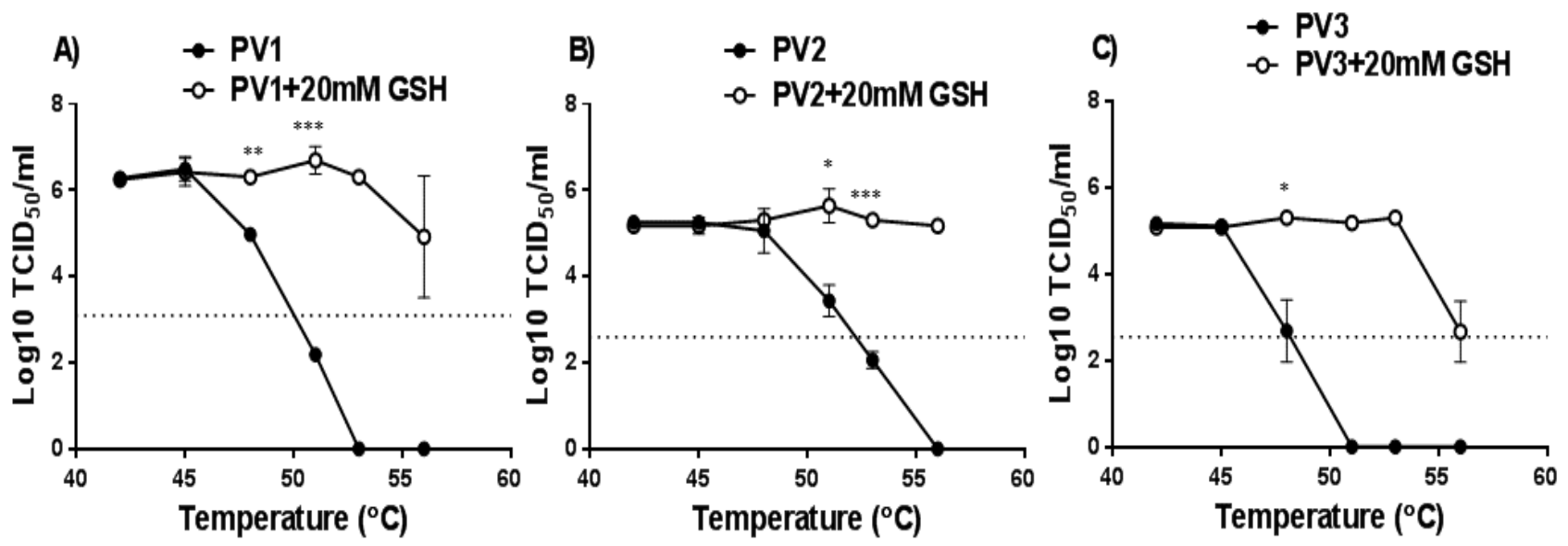

Fig.2. Effect of glutathione on the thermostability profile of polioviruses

Polioviruses types 1 (A), 2 (B) and 3(C) were heat-inactivated for $2 \mathrm{~min}$ at the specified temperatures in presence or absence of $20 \mathrm{mM} \mathrm{GSH}$ after which samples were stored on ice the infectious virus content in each sample was quantified by endpoint titration. The

184 dotted lines represent $50 \%$ of the $\mathrm{TCID}_{50} / \mathrm{ml}$ prior to incubation. Data are the average \pm 185 SD of three independent experiments. Significant differences from the untreated control 186 were determined by the Prism software (GraphPad) using the unpaired Student's t-test 187 $\left(* \mathrm{P}<0.05,{ }^{* *} \mathrm{P}<0.01,{ }^{* * *} \mathrm{P}<0.005\right)$. 

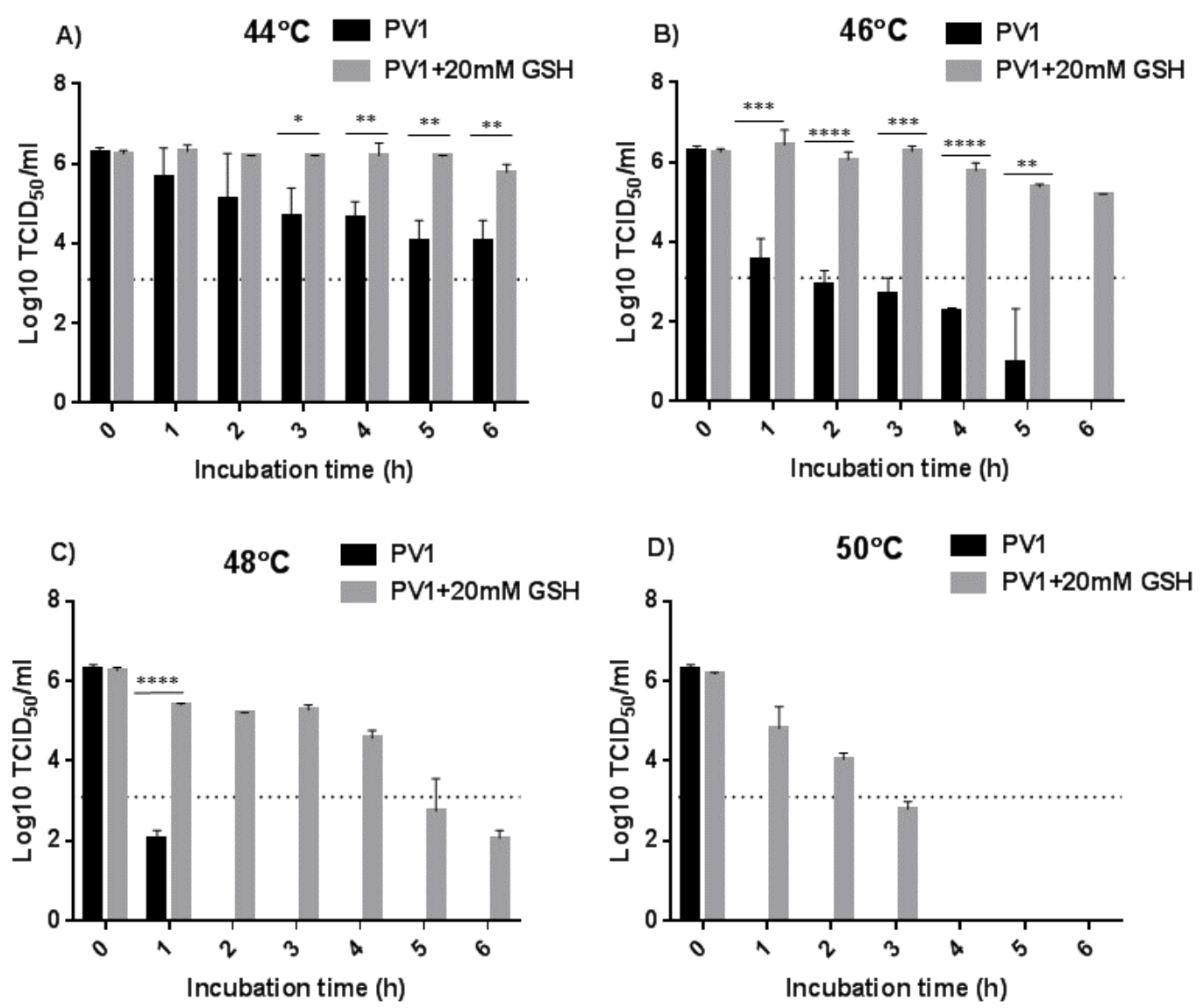

Fig.3. Prolonged heat-stabilization of poliovirus type 1 by GSH

Poliovirus type 1 (PV1) was incubated at either A) $44^{\circ} \mathrm{C}$, B) $46^{\circ} \mathrm{C}, \mathrm{C}$ ) $48^{\circ} \mathrm{C}$ or D) $50^{\circ} \mathrm{C}$ for 6

191 hours in presence or absence of $20 \mathrm{mM}$ GSH. Samples were collected every hour and after cooling on ice, the remaining infectious viral load was determined by endpoint titration. The dotted lines represent $50 \%$ of the $\mathrm{TCID}_{50} / \mathrm{ml}$ prior to incubation. The data are the average \pm SD of three independent experiments. Significant differences from the untreated control were assessed using the unpaired Student's t-test $\left({ }^{*} \mathrm{P}<0.05,{ }^{* *} \mathrm{P}<0.01\right.$, $\left.{ }^{* * *} \mathrm{P}<0.005,{ }^{* * * *} \mathrm{P}<0.001\right)$. Statistical analysis was performed using the Prism software (GraphPad). 